\title{
Molecular structure and free energy landscape for electron transport in the decahaem cytochrome MtrF
}

\author{
Marian Breuer*, Piotr Zarzycki†, Liang Shił, Thomas A. Clarke $\S$, Marcus J. Edwards $\S$, Julea N. Butt $\S$, \\ David J. Richardson $\S$, James K. Fredrickson $\ddagger$, John M. Zachara‡, Jochen Blumberger* and Kevin M. Rosso $\ddagger^{1}$ \\ "Condensed Matter and Materials Physics, Department of Physics and Astronomy, Faculty of Mathematical and Physical Sciences, University College London, \\ Gower Street, London WC1E 6BT, U.K., †Institute of Physical Chemistry, Polish Academy of Sciences, 44/52 Kasprzaka, 01-224 Warsaw, Poland, †Pacific \\ Northwest National Laboratory, 902 Battelle Boulevard, Richland, WA 99352, U.S.A., and §University of East Anglia, Norwich Research Park, Norwich NR4 7T), \\ U.K
}

\begin{abstract}
The free energy profile for electron flow through the bacterial decahaem cytochrome MtrF has been computed using thermodynamic integration and classical molecular dynamics. The extensive calculations on two versions of the structure help to validate the method and results, because differences in the profiles can be related to differences in the charged amino acids local to specific haem groups. First estimates of reorganization free energies $\lambda$ yield a range consistent with expectations for partially solvent-exposed cofactors, and reveal an activation energy range surmountable for electron flow. Future work will aim at increasing the accuracy of $\lambda$ with polarizable forcefield dynamics and quantum chemical energy gap calculations, as well as quantum chemical computation of electronic coupling matrix elements.
\end{abstract}

\section{Introduction}

Dissimilatory metal-reducing bacteria possess the ability to use solid metal oxides such as ferrihydrite and manganese oxide $[1,2]$ as terminal electron sinks. As these substrates are located externally to the cell, the bacteria require a complex network of electron-transfer proteins to transport electrons from the site of substrate oxidation at the inner membrane across the periplasm and the outer membrane to the cell exterior [3]. It is speculated that, in certain cases, these same electron-transfer proteins can also facilitate electron transport over distances of several micrometres, along conductive bacterial pili $[4,5]$, by multi-step charge hopping [6]. Therefore the electron-transfer proteins involved in these processes are interesting not only in their biological context, but also for the promise of nanobiotechnological applications $[7,8]$. The purpose of the present article is to examine the frontier of our understanding in the molecular function of these outer-membrane electron-transfer proteins, and contribute new insights from theory. The recent report of the crystal structure of MtrF [9] has provided the first and only submolecular structural information to date; we therefore focus on the protein MtrF.

$\mathrm{MtrF}$ is a decahaem c-type cytochrome found on the outer membrane of Shewanella oneidensis MR-1; like its homologue $\mathrm{MtrC}$, it is proposed to help facilitate electron transport through the outer membrane to solid substrates,

Key words: $c$-type cytochrome, decahaem cytochrome, electron transport, free energy, MtrF, Shewanello oneidensis.

Abbreviations used: PFV, protein film voltammetry.

${ }^{1}$ To whom correspondence should be addressed (email kevin.rosso@pnnl.gov). in co-operation with a periplasmic cytochrome $\mathrm{MtrD}$ as electron donor and a membrane barrel protein MtrE to enable contact between MtrD and MtrF through the outer membrane (Figure 1). The structure of MtrF consists of four domains, with domains I and III containing $\beta$-sheets and domains II and IV containing $\alpha$-helices. The latter two domains each bind five haem $c$ cofactors, covalently bound to the protein via two cysteine linkages in a $\mathrm{CXXCH}-$ binding motif and ligated by two histidine residues. The haem cofactors are arranged in a 'staggered-cross' formation, with the four roughly co-planar haems 2, 1, 6 and 7 in the centre of the protein and the haem-triples 3,4 and 5 and 8, 9 and 10 being nearly parallel-stacked and placed perpendicular to the central haem plane. This arrangement yields an octahaem chain between haems 5 and 10, with haems 1 and 6 as branching points towards haems 2 and 7 respectively.

Although this structure is suggestive of efficient directional haem-haem electron transfer along multihaem chains, many aspects of the function of the protein are still largely unknown, including the thermodynamics and kinetics of electron transport. Haem redox potentials for solvated MtrF were measured to lie in the range 0 to $-0.260 \mathrm{~V}$, and a set of ten redox potentials in a similar range, -0.044 to $-0.312 \mathrm{~V}$, was obtained from PFV (protein film voltammetry) [9]. Yet, assignment of these values to individual haems is difficult owing to their nearly identical chemical nature. The haemhaem electron transfer free energy 'landscape' along chains in $\mathrm{MtrF}$ thus remained obscure.

Large-scale molecular dynamics simulations employing thermodynamic integration were performed to address this deficiency. In a previous publication [10], the free energy 
Figure 1 | Crystal structure of MtrF and proposed interaction with cytochrome MtrD and barrel protein MtrE

Haems are numbered with Arabic numerals, and domains are numbered with Roman numerals. Iron atoms are emphasized in orange.

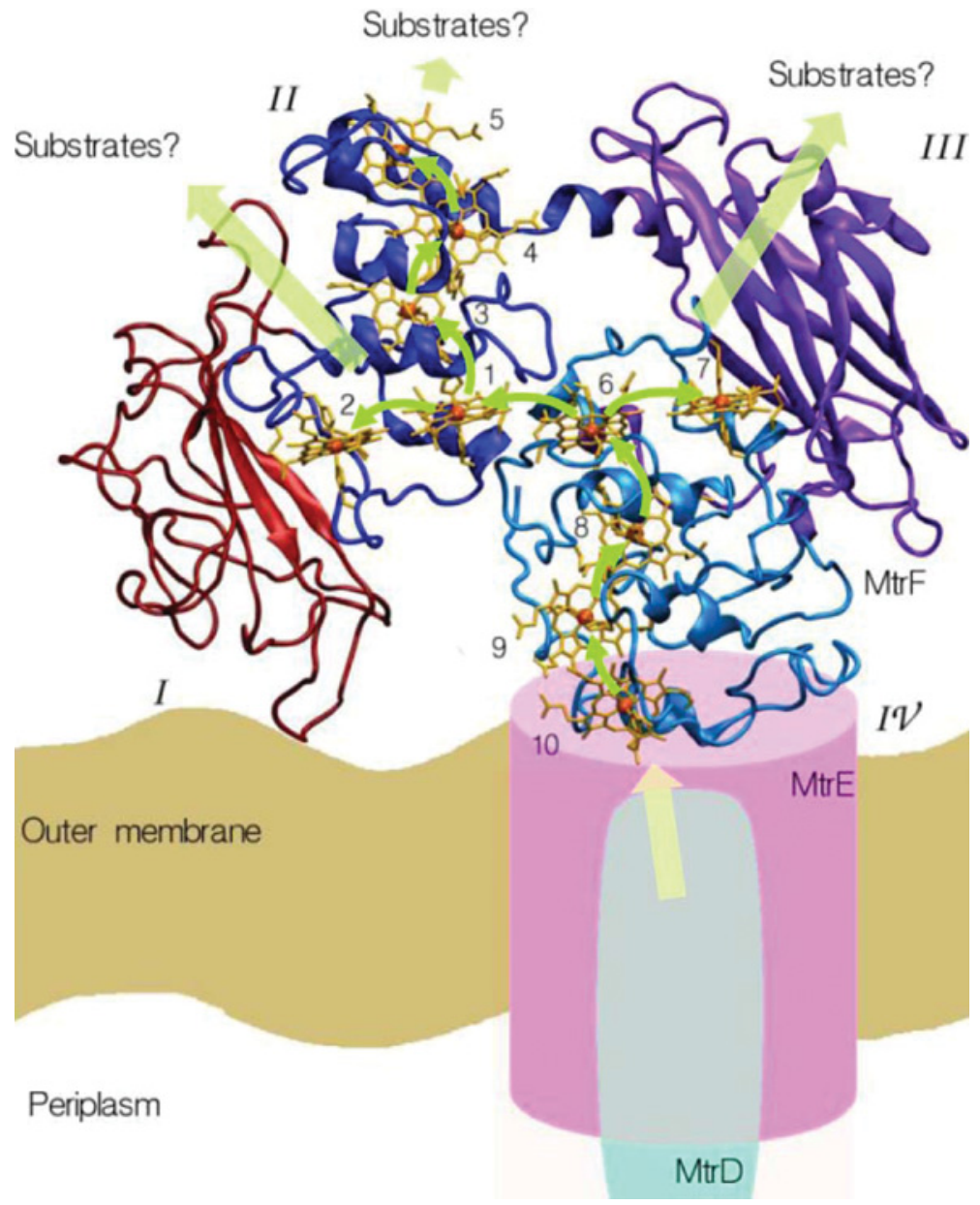

landscape for the published crystal structure of MtrF was presented, featuring an almost symmetrical profile and a range of potentials in good agreement with experimental data (approximately $0.3 \mathrm{~V}$ compared with $0.26 \mathrm{~V}$ in [9]). In the present paper, we describe additional related simulation work to provide new insights into the relationship between structure and the free energy of electron transfer along haem chains, by comparing the published simulations with those performed on a preliminary incomplete version of the MtrF structure (T.A. Clarke and M.J. Edwards, unpublished work). The preliminary structure was selected as an ideal alternative for comparison because it conveniently provides a random selection of structure differences for which effects on the free energy landscape can be assessed. Figure 2 shows the final MtrF structure emphasizing differences with the preliminary structure.

Among residue absences or differences as indicated, of particular importance are charged residues, which can strongly influence haem redox potentials. For example, among those indicated, the green loop in the left half of Figure 2 shows a group of 11 residues present in the final structure, but which are lacking in the preliminary structure, one of them being a glutamate residue $\left(\mathrm{Glu}^{109}\right.$; the other new residues are uncharged). Another new residue (threonine) occurs in the neighbourhood of haem 10. Changed residues furthermore introduce a glutamate residue $\left(\mathrm{Glu}^{618}\right)$ close to haem 10, an arginine residue ( $\mathrm{Arg}^{207}$ ) below haem 2 and another arginine residue $\left(\mathrm{Arg}^{267}\right)$ in the vicinity of haem 4 . All other changes did not introduce or remove any charges. The final crystal structure also features a $\mathrm{Ca}^{2+}$ ion located on the protein surface in the neighbourhood of haem 3 which was found to be mobile during the simulations, however.

Comparison of simulation results on the two structures provides us with a much larger dataset and enables us to relate differences in the sets of redox potentials to random changes in residues and local protein configuration between the preliminary and final MtrF. We also use the preliminary structure to compare two different thermodynamic integration pathways, thus enabling investigation of the free energy profile sensitivity to the integration pathway chosen (see the Supplementary Online Data at http://www. biochemsoctrans.org/bst/040/bst0401198add.htm). Within 
Figure 2 | Differences between the final and preliminary MtrF structures (PDB code 3PMQ)

The image shows the final structure with all new or changed residues highlighted: green for new residues, cyan for changed residues. Charged residues not present in the preliminary structure are highlighted in red (negative) or blue (positive)

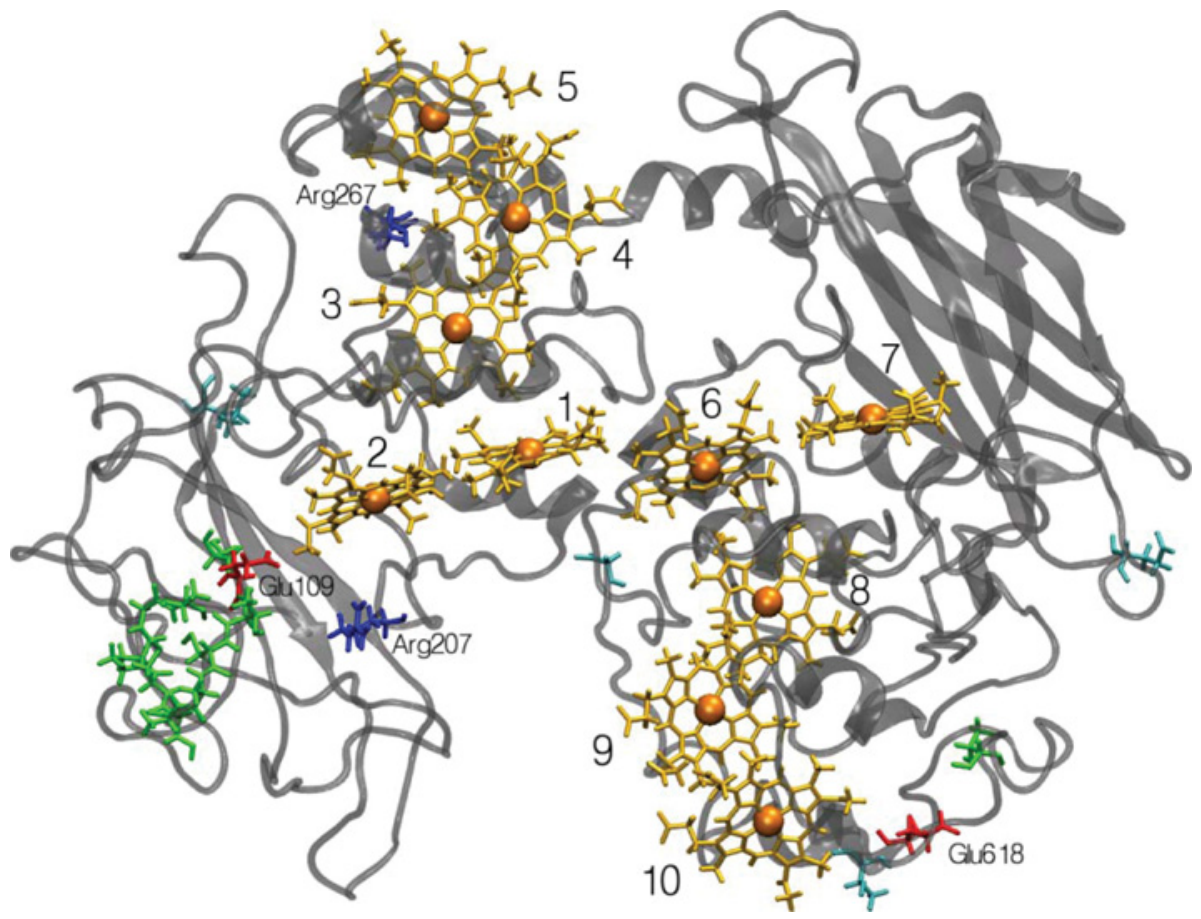

the framework of the linear response approximation, the molecular dynamics trajectories were also used to extract first estimates of reorganization free energies for electron transfer between adjacent haems in the final structure. These new data enable the construction of the free energy barriers for haemhaem electron transfer along the free energy profile for MtrF.

\section{Structure effects on the free energy profile}

Figure 3(a) shows the free energy profiles obtained via thermodynamic integration (integration pathway: oxidation of individual cofactors) for the preliminary and final MtrF structures [10] (see Supplementary Table S1 at http://www.biochemsoctrans.org/bst/040/bst0401198add. htm for the numerical values of the redox potentials). Both profiles comprise the averages of forward and backward integration for each haem, and show half of the difference as uncertainty; these uncertainties are typically not larger than $\pm 0.03 \mathrm{~V}$. The uncertainties in the individual directions resulting from finite simulation length were always smaller than $0.01 \mathrm{~V}$ and thus considered insignificant compared with the difference for forward/backward simulation. Both sets of redox potentials span virtually the same range $(0.35 \mathrm{~V}$ for the final structure, and $0.36 \mathrm{~V}$ for the preliminary structure). The values for haems 9 and 5 for each structure lie within the uncertainty range of the other structure's value for that haem; similarly, for haems 10, 1 and 3, the error bars nearly overlap. Haems 8, 6 and 7 show higher potentials in the final structure, whereas haems 2 and 4 show lower potentials in the final structure. Despite the differences, the eye-catching feature of the free energy hill 10-9-8-6 is qualitatively well conserved between the two, and the relative potentials of haems 8, 6 and 7 are conserved within the present uncertainty ranges. The largest differences are observed for haems 2 and 4 which both show a significant fall in potential in the final structure. Nonetheless, haem 4 does constitute a small free energy hill in the preliminary structure as well.

Although it is impossible to fully deconvolute the observed potential differences into contributions of individual residues and ions, we can highlight a few significant differences. Most of these occur in the neighbourhood of haems 10, 2, 3 and 4 , i.e. in the region that also shows a qualitative change in the free energy profile between the two structures. The most obvious change is the additional Glu ${ }^{618}$ in the final structure (Figure 2). In the light of this additional negative charge in close proximity to haem $10[14 \AA(1 \AA=0.1 \mathrm{~nm})$ distance between iron and carboxy-oxygen in the crystal structure], a potential fall for haem 10 as seen in Figure 3(a) is plausible. In the proximity of haem 2 , the final structure includes two new residues of opposite charges, $\mathrm{Glu}^{109}$ and $\mathrm{Arg}^{207}$, at a similar distance to haem 2 (both around $9 \AA$ to the iron in the crystal structure). However, $\mathrm{Asp}^{228}$ has a different position in the two structures, being much closer to haem 2 in the final structure (see Supplementary Figure S2 at http://www. biochemsoctrans.org/bst/040/bst0401198add.htm). During the dynamics, its carboxy group maintains a distance of 
Figure 3 | Free energy data for electron transfer through MtrF

(a) Free energy profiles for the final and preliminary structures of MtrF. Values are averages for forward and backward thermodynamic integrations (oxidation pathway) with their differences as uncertainties. (b) Calculated Marcus free energy parabolas for electron transfer between adjacent haems in MtrF (final structure). Haems 1-10 are numbered.
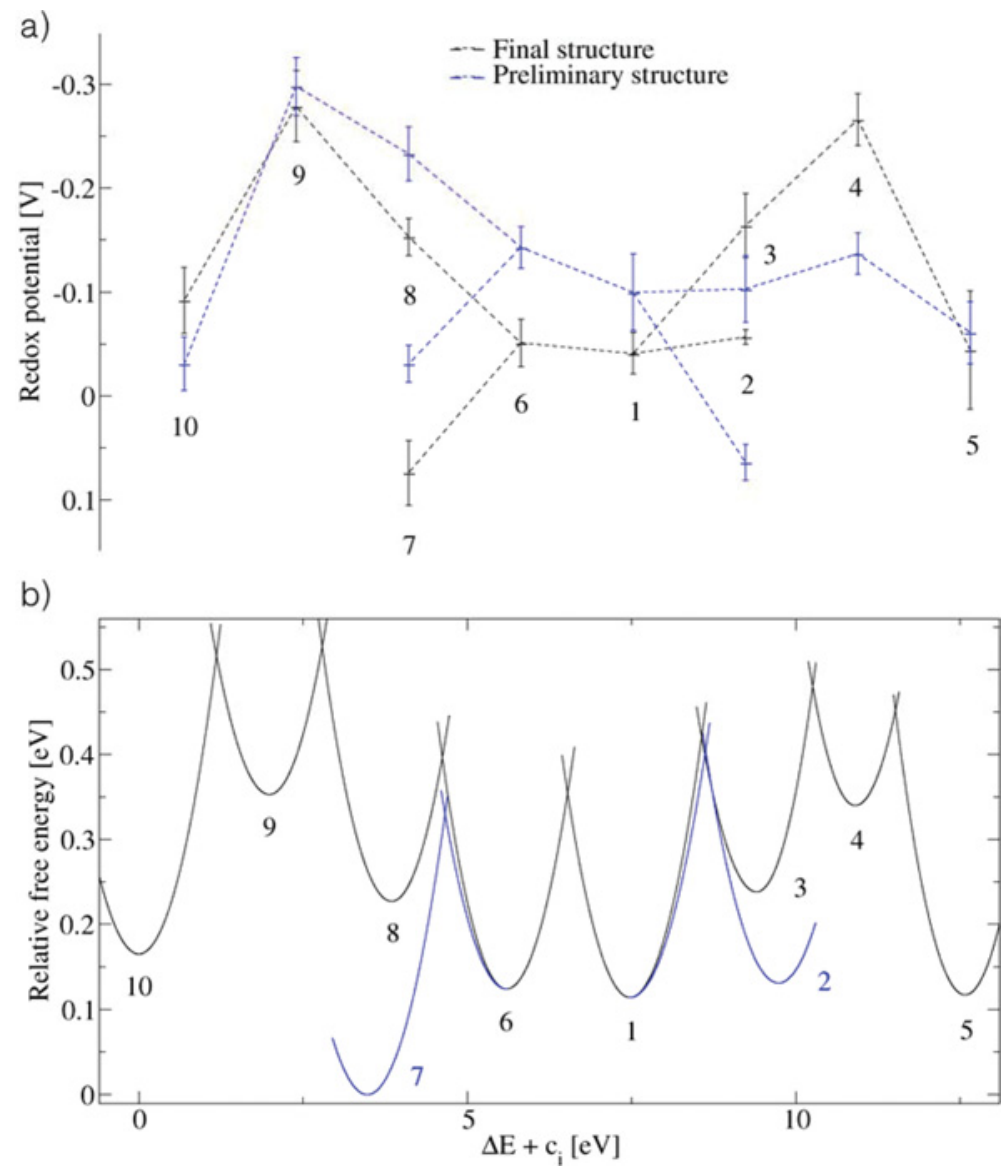

approximately $2.5 \AA$ to the adjacent histidine ligand most of the time. In addition, in its position in the preliminary structure, the aspartate residue forms an ion-binding site together with a glutamate $\left(\mathrm{Glu}^{272}\right.$; see Supplementary Figure S3 at http://www.biochemsoctrans.org/bst/040/ bst0401198add.htm), binding a cation during the dynamics with a distance of approximately $11 \AA$ to haem 3, $13 \AA$ to haem 4 and $17 \AA$ to haem 2. Finally, we observe a similar formation of an ion-binding site in the preliminary structure in the proximity of haem 4 , involving an aspartate residue $\left(\mathrm{Asp}^{431}\right)$ which, during the dynamics, turns towards $\mathrm{Asn}^{309}$ and $\mathrm{Ser}^{312}$, forming an ion-binding site with their backbone carbonyl groups (see Supplementary Figure S4 at http://www.biochemsoctrans.org/bst/040/bst0401198add. htm; however, this site is less stable than the other one and dissolves in between). In forming this site, $\mathrm{Asp}^{431}$ has to move away from its crystal structure position; however, this was not observed for the final structure which generally featured lower RMSD (root mean square deviation) values, and the ion affinity of $\mathrm{Asn}^{309}$ and $\mathrm{Ser}^{312}$ alone was much lower. The large change observed in the potential of haem 2 can be rationalized by the close proximity of $\mathrm{Asp}^{228}$ in the final structure. For haems 3 and 4 , the absence of close-by ion-binding sites in the final structure provides a possible reason for these haems' potentials decreasing from the preliminary to the final structure, despite the addition of a positively charged residue $\left(\mathrm{Arg}^{267}\right)$ in their neighbourhood (see Figure 2). Thus, overall, this comparative analysis suggests that it is the nature of the local charged groups that probably defines the free energy landscape in MtrF.

\section{Reorganization free energies}

With reference hereinafter only to the final structure of MtrF, the reorganization free energies $\lambda$ as obtained from the molecular dynamics trajectories are listed in Table 1, together with the resulting activation free energies $\Delta E^{\ddagger}$. Figure $3(\mathrm{~b})$ shows the resulting Marcus free energy parabolas for electron transfer. The values for $\lambda$ cover the range from 0.75 to 1.1 $\mathrm{eV}$; values for $\Delta E^{\ddagger}$ span a range of approximately $250 \mathrm{meV}$. The reorganization free energies $\lambda$ reported in Table 1 were obtained using a polarizable forcefield to calculate vertical 
Table 1 | Reorganization free energies $\lambda$ and activation free energies $\Delta \boldsymbol{E}^{\ddagger}$ for the final structure of MtrF

Forward and backward for pair $x y$ refers to $\Delta E^{\ddagger}$ for $x \rightarrow y$ and $y \rightarrow x$ respectively.

\begin{tabular}{llll}
\hline Pair & $\lambda(\mathrm{eV})$ & $\Delta \boldsymbol{E}^{\ddagger}$ forward $(\mathrm{eV})$ & $\Delta \boldsymbol{E}^{\ddagger}$ backward $(\mathrm{eV})$ \\
\hline $1 \rightarrow 2$ & 1.13 & 0.29 & 0.27 \\
$1 \rightarrow 3$ & 0.96 & 0.31 & 0.18 \\
$1 \rightarrow 6$ & 0.94 & 0.24 & 0.23 \\
$3 \rightarrow 4$ & 0.75 & 0.24 & 0.14 \\
$4 \rightarrow 5$ & 0.84 & 0.11 & 0.34 \\
$6 \rightarrow 7$ & 1.06 & 0.21 & 0.33 \\
$6 \rightarrow 8$ & 0.87 & 0.27 & 0.17 \\
$8 \rightarrow 9$ & 0.93 & 0.30 & 0.17 \\
$9 \rightarrow 10$ & 0.99 & 0.16 & 0.35 \\
\hline
\end{tabular}

electron transfer energies ('energy gaps') on snapshots from the molecular dynamics which were carried out with a non-polarizable force field (see the Supplementary Online Data); this approach does not account fully yet for electronic polarization. Furthermore, an estimated value of $50 \mathrm{meV}$ was used for the inner shell contribution (of the two cofactors involved in electron transfer) in lieu of quantum mechanical inner sphere energy gap calculations (to account for electronic structure changes during electron transfer). The numbers presented thus only constitute first estimates. However, electron transfer between cofactors excluded from the solvent is typically expected to show reorganization energies from 0.25 to $0.9 \mathrm{eV}[11,12]$, whereas values from 0.9 to $1.5 \mathrm{eV}$ are expected if one cofactor is solvent-exposed [11,13,14]. Haems in $\mathrm{MtrF}$ display a relatively large solvent exposure on average [9]. The range computed of $0.75-1.1 \mathrm{eV}$ is therefore rather consistent with expectations.

\section{Implications for electron conductance in MtrF}

The two potential hills in the profile are a quite peculiar feature; the question arises why evolution should introduce upslope electron-transfer steps rather than simply employing a flat free energy landscape with a continuous downhill slope. One consideration is that a continuous downhill slope would suffer the disadvantage of dissipating $0.26 \mathrm{eV}$ of free energy, whereas the calculated profile shows that the net driving force along the octahaem chain from haem 10 to 5, for example, is negligible. Another consideration is that it is also not yet known how much the uphill steps actually decrease the total electron-transport rate through MtrF. For example, the estimated activation free energies $\Delta E^{\ddagger}$ for the electrontransfer steps $1 \rightarrow 2$ and $1 \rightarrow 3$ are almost the same (see Table 1 ) despite the redox potential of haem 3 being approximately $0.1 \mathrm{~V}$ lower than the potentials of haem 1 and 2, owing to the smaller reorganization energy for haem $1 \rightarrow 3$ electron transfer. Furthermore, nothing is known yet about electronic coupling matrix elements, the respective values for which could reorder haem-haem electron-transfer rates along the chains. Thus the two free energy hills in the profile need not necessarily constitute an electron-conductance problem.

With regard to possible electron-transfer functions of the two low-potential haems 4 and 9, we noted previously [10] that these would be the only ones capable of spontaneously reducing the soluble electron shuttle FMN (redox potential $-219 \mathrm{mV}$ [15]), shown experimentally to be a feasible substrate for MtrF [9]. That only a subgroup of the ten haems is calculated to be thermodynamically favourable for FMN reduction is broadly consistent with the experimental data that showed that FMN did not fully oxidize the reduced MtrF protein, but only oxidized it by approximately $30-40 \%$ [9]. EPR and PFV analyses also showed that only $30-40 \%$ of the haems are oxidized at approximately $-200 \mathrm{mV}$ [9]. However, addition of $\mathrm{Fe}$ (III) citrate to reduced MtrF fully oxidizes all ten haems, which is consistent with the $E_{0}$ ' of $\mathrm{Fe}(\mathrm{III}) / \mathrm{Fe}$ (II) being $>100 \mathrm{mV}$ (i.e. exceeding the highest computed value for haem 7; see Figure 3) [9]. Even though haems 4 and 9 are not terminal haems of a chain, their reported solvent-exposed surface areas are just as high as those of haems 2 and 7, the putative exit/entrance points of the tetrahaem chain [9]. Thus their non-terminal positions may not exclude their redox interaction with a soluble molecule such as FMN. The question then arises whether there might be a strategic advantage to have the most energetically favourable FMN-reduction sites inside the chain rather than at terminal haems. One advantage might be that they could still be sterically accessible even when MtrF has docked to a solid substrate or another cytochrome. For example, if $\mathrm{MtrF}$ were to comprise a repeating electron-transfer conduit along conductive pili (whose precise constituents and their arrangement so far remain unknown), and supposing that haems 10 and 5 were contact points to receive and pass on electrons to adjacent cytochromes, the 'sideways' chains consisting of the tetrahaem chain 2, 1, 6 and 7 might then collectively provide a large contact area for a solid substrate (certainly larger than that of haem 5 or 10 alone). If haems 10 and 5 were forming contacts with partner proteins and haems 2, 1, 6 and 7 were making contact with a solid substrate, haems 4 and 9 would be the only cofactors left physically accessible for a soluble substrate (as haems 3 and 8 are relatively buried in the protein). In this way, eight out of ten haems could be involved in electron-transfer processes with various soluble and insoluble redox partners.

The free energy profile for electron flow through the bacterial decahaem cytochrome MtrF has been computed using thermodynamic integration and classical molecular dynamics. The extensive calculations on two versions of the structure help to validate the method and results, because differences in the profiles can be related to differences in the charged amino acids local to specific haem groups. First estimates of reorganization free energies $\lambda$ yield a range consistent with expectations for partially solventexposed cofactors, and reveal an activation energy range surmountable for electron flow. Future work will aim to increase the accuracy of $\lambda$ with polarizable forcefield dynamics and quantum chemical energy gap calculations, as 
well as quantum chemical computation of electronic coupling matrix elements.

\section{Acknowledgements}

This work was carried out on HECToR, the U.K.'s national highperformance computing service, access to which was granted via the Materials Chemistry Consortium (Engineering and Physical Sciences Research Council grant EP/F067496), on the supercomputer Chinook, based at the Environmental Molecular Sciences Laboratory at Pacific Northwest National Laboratory (PNNL), and on the University College London (UCL) Legion High Performance Computing Facility.

\section{Funding}

M.B. gratefully acknowledges an IMPACT studentship co-sponsored by University College London (UCL) and Pacific Northwest National Laboratory (PNNL) through the U.S. Department of Energy's Subsurface Biogeochemistry Research Science Focus Area (SBR-SFA) programme of the Office of Biological and Environmental Research. The Office of Biological and Environmental Research also provided support for K.M.R. and P.Z. J.B. thanks the Royal Society for a University Research Fellowship. P.Z. also thanks the European Union Framework Programme 7 for support by NOBLESSE grant [grant number REGPOT-2011-1]

\section{References}

1 Myers, C.R. and Nealson, K.H. (1988) Baterial manganese reduction and growth with manganese oxide as the sole electron-acceptor. Science 240, 1319-1321

2 Straub, K.L., Benz, M. and Schink, B. (2001) Iron metabolism in anoxic environments at near neutral pH. FEMS Microbiol. Ecol. 34, 181-186

3 Hartshorne, R.S., Reardon, C.L., Ross, D., Nuester, J., Clarke, T.A., Gates, A.J., Mills, P.C., Fredrickson, J.K., Zachara, J.M., Shi, L. et al. (2009) Characterization of an electron conduit between bacteria and the extracellular environment. Proc. Natl. Acad. Sci. U.S.A. 106, 22169
4 El-Naggar, M.Y., Wanger, G., Leung, K.M., Yuzvinsky, T.D., Southam, G., Yang, J., Lau, W.M., Nealson, K.H. and Gorby, Y.A. (2010) Electrical transport along bacterial nanowires from Shewonello oneidensis MR-1. Proc. Natl. Acad. Sci. U.S.A. 107, 18127-18131

5 Gorby, Y.A., Yanina, S., McLean, J.S., Rosso, K.M., Moyles, D., Dohnalkova, A., Beveridge, T.J., Chang, I.S., Kim, B.H., Kim, K.S. et al. (2006) Electrically conductive bacterial nanowires produced by Shewonello oneidensis strain MR-1 and other microorganisms. Proc. Natl. Acad. Sci. U.S.A. 103, 11358-11363

6 Polizzi, N.F., Skourtis, S.S. and Beratan, D.N. (2012) Physical constraints on charge transport through bacterial nanowires. Faraday Discuss. 155, 43-62

7 Xiong, Y.J., Shi, L., Chen, B.W., Mayer, M.U., Lower, B.H., Londer, Y., Bose, S., Hochella, M.F., Fredrickson, J.K. and Squier, T.C. (2006) High-affinity binding and direct electron transfer to solid metals by the Shewonella oneidensis MR-1 outer membrane c-type cytochrome OmcA. J. Am. Chem. Soc. 128, 13978-13979

8 Jensen, H.M., Albers, A.E., Malley, K.R., Londer, Y.Y., Cohen, B.E., Helms, B.A., Weigele, P., Groves, J.T. and Ajo-Franklin, C.M. (2010) Engineering of a synthetic electron conduit in living cells. Proc. Natl. Acad. Sci. U.S.A. 107, 19213-19218

9 Clarke, T.A., Edwards, M.J., Gates, A.J., Hall, A., White, G.F., Bradley, J., Reardon, C.L., Shi, L., Beliaev, A.S., Marshall, M.J. et al. (2011) Structure of a bacterial cell surface decaheme electron conduit. Proc. Natl. Acad. Sci. U.S.A. 108, 9384-9389

10 Brever, M., Zarzycki, P., Blumberger, J. and Rosso, K.M. (2012) Thermodynamics of electron flow in the bacterial deca-heme cytochrome MtrF. J. Am. Chem. Soc. 134, 9868-9871

11 Moser, C.C., Page, C.C. and Dutton, P.L. (2006) Darwin at the molecula scale: selection and variance in electron tunnelling proteins including cytochrome c oxidase. Philos. Trans. R. Soc. London Ser. B $\mathbf{3 6 1}$ 1295-1305

12 Tipmanee, V. and Blumberger, J. (2012) Kinetics of the terminal electron transfer step in cytochrome c oxidase. J. Phys. Chem. B 116, 1876-1883

13 Tipmanee, V., Oberhofer, H., Park, M., Kim, K.S. and Blumberger, J. (2010) Prediction of reorganization free energies for biological electron transfer: a comparative study of ru-modified cytochromes and a 4-helix bundle protein. J. Am. Chem. Soc. 132, 17032-17040

14 Blumberger, J. (2008) Free energies for biological electron transfer from QM/MM calculation: method, application and critical assessment. Phys. Chem. Chem. Phys 10, 5651-5667

15 Brown, J.P. (1981) Reduction of polymeric azo and nitro dyes by intestinal bacteria. Appl. Environ. Microbiol. 41, 1283-1286

Received 1 June 2012

doi:10.1042/BST20120139 
SUPPLEMENTARY ONLINE DATA

\title{
Molecular structure and free energy landscape for electron transport in the decahaem cytochrome MtrF
}

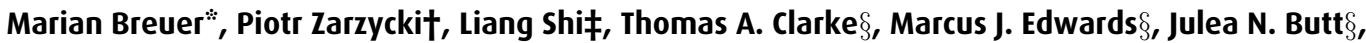 \\ David J. Richardson $\S$, James K. Fredricksonł, John M. Zacharał, Jochen Blumberger* and Kevin M. Rossoł \\ "Condensed Matter and Materials Physics, Department of Physics and Astronomy, Faculty of Mathematical and Physical Sciences, University College London, \\ Gower Street, London WC1E 6BT, U.K., †Institute of Physical Chemistry, Polish Academy of Sciences, 44/52 Kasprzaka, 01-224 Warsaw, Poland, †Pacific \\ Northwest National Laboratory, 902 Battelle Boulevard, Richland, WA 99352, U.S.A., and §University of East Anglia, Norwich Research Park, Norwich NR4 7T), \\ U.K.
}

\section{Computational method}

Redox potentials were calculated for both preliminary and final structure using classical molecular dynamics and thermodynamic integration where the free energy difference between two states (denoted by 0 and 1 ) is given by:

$$
\Delta G=\int_{0}^{1}\left\langle\frac{\partial E_{\eta}}{\partial_{\eta}}\right\rangle_{\eta} d \eta
$$

where $\frac{\partial E_{\eta}}{\partial_{\eta}}=E_{1}-E_{0}+(2 \eta-1) E_{\Delta}, E_{1}$ and $E_{0}$ are the potential energies in the final and initial state respectively, and $E_{\Delta}$ is an additional contribution due to the integration path chosen between the initial and final state (linear charge morphing rather than linear energy morphing). The angular brackets denote an ensemble average over the state given by $\eta$. The electron-supply-limited case was considered, so that all cofactors bar one were in the oxidized state. Two different free energies were computed: free energies of oxidation of individual cofactors (for both final and preliminary structure), yielding redox potentials $\varepsilon$ via $\Delta G=F \varepsilon$, where $F$ is the Faraday constant; and free energies of electron transfer between adjacent haems (preliminary structure only), yielding relative free energies and thus relative redox potentials. In each case, integrations were carried out in both directions $(0 \rightarrow 1$ and $1 \rightarrow 0)$ to obtain average values. All thermodynamic integration simulations were carried out using the AMBER03 forcefield (http://ambermd.org/) in combination with haem parameters as reported in previous studies [1-3]. As $\frac{\partial E_{\eta}}{\partial_{\eta}}$ was calculated in periodic boundary conditions and with classical point charges for the cofactors, computed absolute redox potentials are offset relative to experiment by a (haem-independent) constant $\mathrm{C}$ which was determined for each set by minimizing the offset between computed and experimental redox potentials (from PFV). The detailed protocol for the simulations and thermodynamic integration on the final structure is given in our previous

${ }^{1}$ To whom correspondence should be addressed (email kevin.rosso@pnnl.gov). paper [4]; the protocol used for the preliminary structure was essentially the same.

The trajectories with fully reduced cofactors were used to calculate reorganization energies $\lambda$ for electron transfer between adjacent cofactors via the linear response approximation, i.e. $\lambda=\frac{\langle\Delta E\rangle_{0}-\langle\Delta E\rangle_{1}}{2}$ [3], where 0 and 1 denote the initial and final state of the electron-transfer step (i.e. the electron being located on the donor or acceptor) respectively and $\Delta E$ is equal to the vertical electron-transfer energy gap $E_{1}-E_{0}$ (the angular brackets again indicating ensemble averages, here over the initial and final state). For the calculation of $\Delta E$ for every snapshot, the polarizable AMBER02 forcefield was used. The contribution of the inner sphere (i.e. atoms involved in the charge change) was subtracted to obtain the outer sphere contribution only; in lieu of quantum chemical inner sphere energy gap calculations, an inner sphere contribution estimate of $50 \mathrm{meV}$ was added to the obtained outer sphere contribution to obtain an estimate for the total reorganization free energy.

\section{Oxidation compared with electron transfer}

Figure S1 shows the free energy profiles obtained for the preliminary structure using the two thermodynamic integration pathways, oxidation and electron transfer (see the Computational method section above). As one haem had to be used as a reference point arbitrarily in constructing the free energy profile for the electron transfer integration ('electrontransfer profile' below), no uncertainties are available for the latter. The electron-transfer profile spans a range of $0.3 \mathrm{~V}$, in better agreement with experiment than the profile from the oxidation pathway ('oxidation profile') with $0.36 \mathrm{~V}$. Figure S1 shows that the two profiles agree very well, with the electrontransfer-derived potentials of half of the haems $(8,7,3,4$ and 5) falling within the uncertainty range of their oxidationderived counterpart and the potential of haem 6 almost lying within the oxidation-derived uncertainty range. The potential of haem 1 is not far off either: its uncertainty range would 
Figure S1 | Free energy profiles for the preliminary structure as obtained via two different thermodynamic integration pathways The two pathways were oxidation of a single cofactor and electron transfer between two cofactors. Both sets of results are averages for forward and backward integrations, and uncertainties for the oxidation profile are half of the difference between forward/backward potential values. Haems 1-10 are numbered.

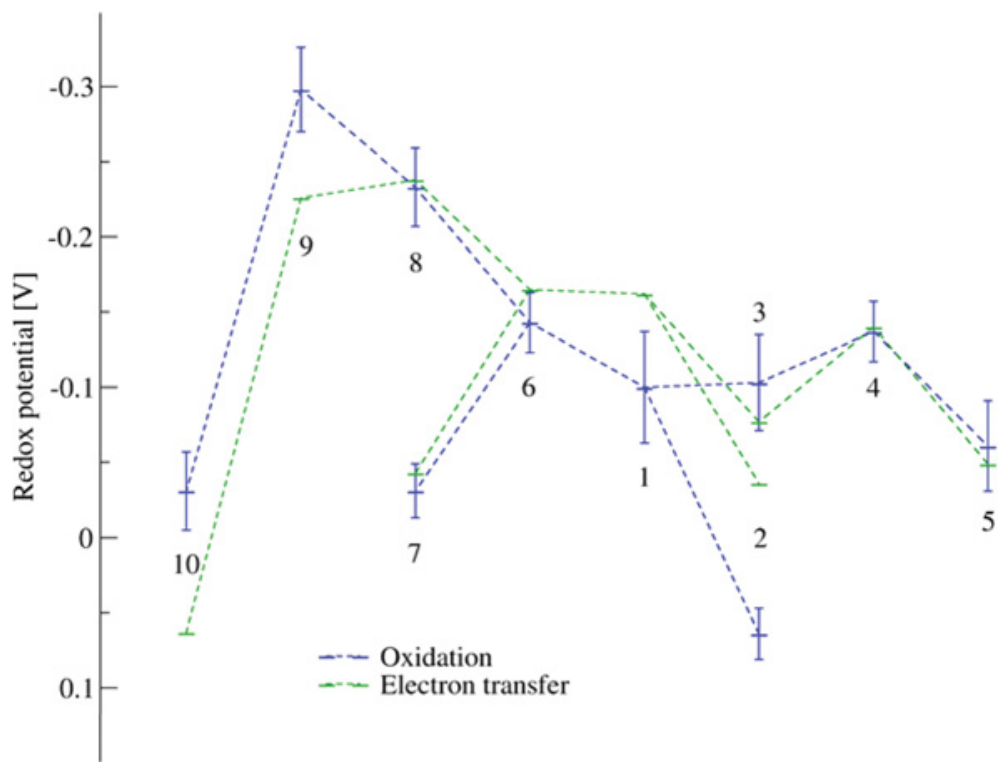

overlap with its oxidation-derived counterpart if one were to assume a similar uncertainty. Haems 10 and 9 both have a slightly higher potential than in the oxidation profile, but their potential difference stays almost the same (change by $0.02 \mathrm{~V}$ ). Thus the left end of the electron-transfer profile is still in very good qualitative agreement with the oxidation profile. The only more pronounced deviation between the two profiles occurs for haem 2 whose potential decreases by approximately $0.11 \mathrm{~V}$ with respect to the oxidation profile.

The profiles in Figure S1 confirm that the thermodynamic integration results at large are insensitive to the choice of integration path as haem 2 is the only cofactor to show pronounced deviation between the two profiles. This insensitivity to the chosen integration pathway serves as justification for the use of only one integration pathway for the final structure. Conversely, as the two pathways yield essentially the same free energy profile, this strengthens trust in the profile itself for the preliminary structure and, on the basis of the comparison in the main text, in the profile for the final structure as well.
Figure S2 | Different positions of Asp $^{228}$ in preliminary and final structure

The upper position of Asp ${ }^{228}$ stems from the preliminary structure, the lower position (closer to haem 2) arises from the final structure.

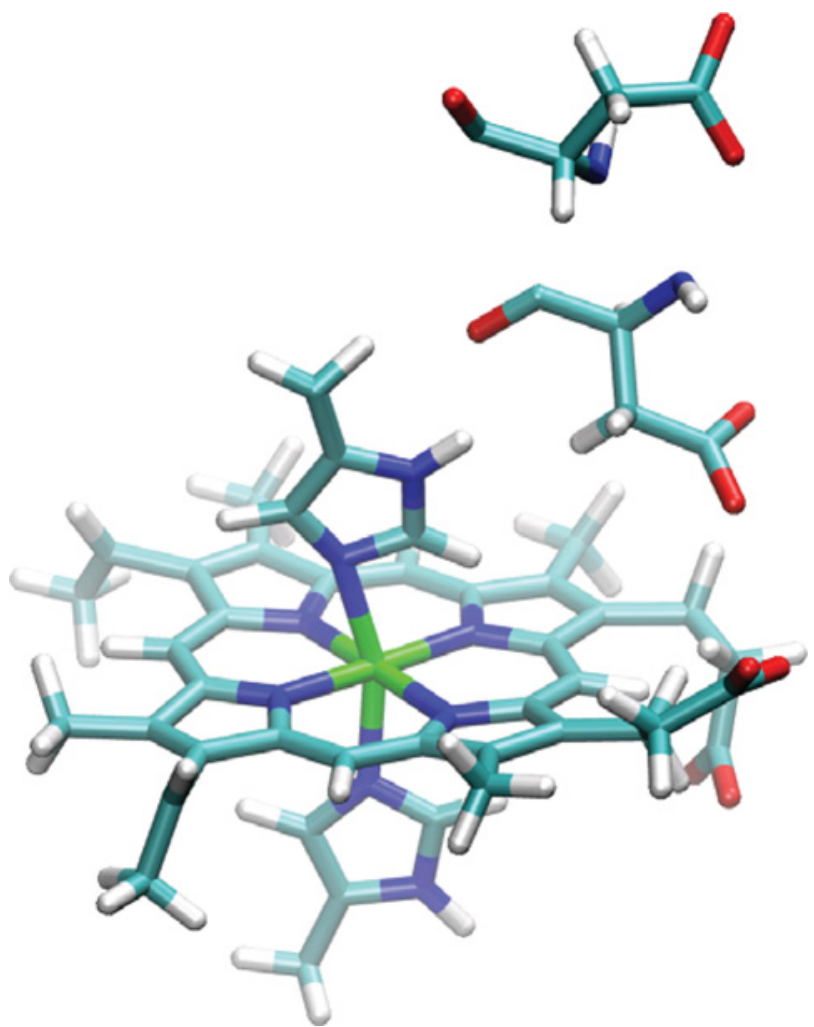


Figure S3 | Ion-binding site formed by $\mathrm{Asp}^{228}$ and Glu $\mathrm{u}^{272}$ in the preliminary structure

The bound cation is shown in blue.

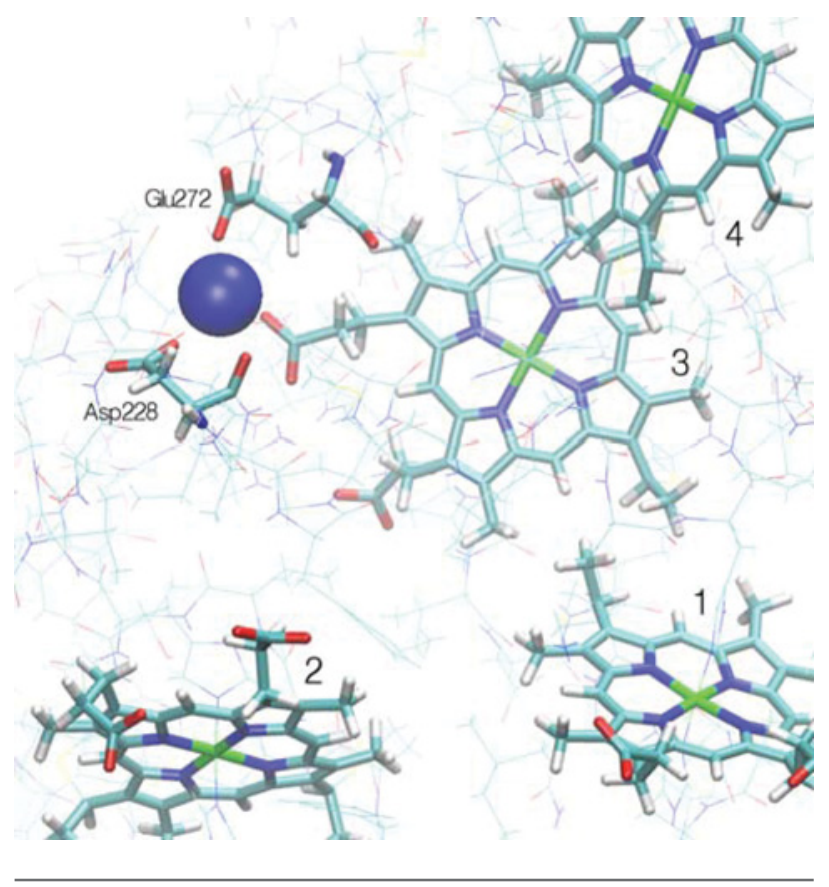

Figure S4 | Ion-binding site formed by $\mathrm{Asn}^{309}$, $^{3 e r^{312}}$ and Asp 431 in the preliminary structure

The bound cation is shown in blue.

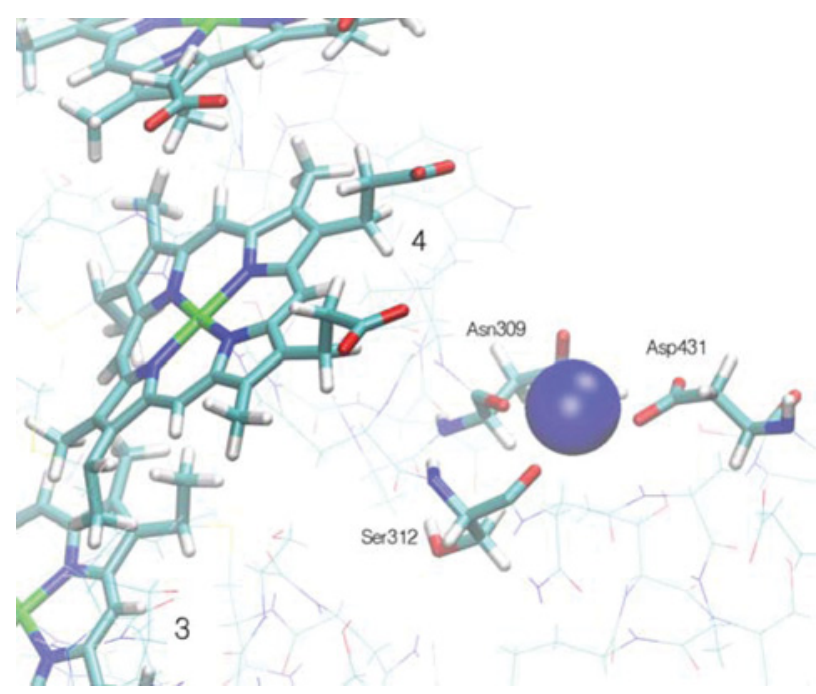

Table S1 | Redox potentials for each haem in the initial and final structure, integration pathway: oxidation of a single cofactor

Values are averages of integration in both directions, with half of the difference as uncertainties.

\begin{tabular}{lll}
\hline & \multicolumn{2}{l}{ Redox potential $(\mathrm{V})$} \\
\cline { 2 - 3 } Haem & Final structure & Preliminary structure \\
\hline 1 & $-0.04 \pm 0.02$ & $-0.10 \pm 0.04$ \\
2 & $-0.06 \pm 0.01$ & $0.06 \pm 0.02$ \\
3 & $-0.16 \pm 0.03$ & $-0.10 \pm 0.03$ \\
4 & $-0.27 \pm 0.03$ & $-0.14 \pm 0.02$ \\
5 & $-0.04 \pm 0.06$ & $-0.06 \pm 0.03$ \\
6 & $-0.05 \pm 0.02$ & $-0.14 \pm 0.02$ \\
7 & $0.07 \pm 0.03$ & $-0.03 \pm 0.02$ \\
8 & $-0.15 \pm 0.02$ & $-0.23 \pm 0.03$ \\
9 & $-0.28 \pm 0.03$ & $-0.30 \pm 0.03$ \\
10 & $-0.09 \pm 0.03$ & $-0.03 \pm 0.03$ \\
\hline
\end{tabular}

\section{References}

1 Tipmanee, V. and Blumberger, J. (2012) Kinetics of the terminal electron transfer step in cytochrome c oxidase. J. Phys. Chem. B 116, 1876-1883

2 Tipmanee, V., Oberhofer, H., Park, M., Kim, K.S. and Blumberger, J. (2010) Prediction of reorganization free energies for biological electron transfer: a comparative study of ru-modified cytochromes and a 4-helix bundle protein. J. Am. Chem. Soc. 132, 17032-17040

3 Blumberger, J. (2008) Free energies for biological electron transfer from QM/MM calculation: method, application and critical assessment. Phys. Chem. Chem. Phys. 10, 5651-5667

4 Breuer, M., Zarzycki, P., Blumberger, J. and Rosso, K.M. (2012) Thermodynamics of electron flow in the bacterial deca-heme cytochrome MtrF. J. Am. Chem. Soc. 134, 9868-9871

Received 1 June 2012

doi:10.1042/BST20120139 